\title{
Block method for third order ordinary differential equations
}

\begin{abstract}
The problem of third order ordinary differential equations (ODEs) is solved directly by using the block backward differentiation formula. The block method is constructed by utilizing three back values and by differentiating the interpolating polynomial once, twice and thrice. Two approximated solutions are generated concurrently for each integration step. Numerical results indicate the efficiency of the direct method than the usual approach of transforming it into the first order ODEs.
\end{abstract}

Keyword: Block method; Third order ordinary differential equations (ODEs) 